\title{
Expression of Alpha-Enolase 1 (EN01) in Villi from Patients with Recurrent Miscarriage and Its Effect on Proliferation and Invasion of Villous Trophoblasts
}

Huai-Yun Tang

Lianyungang Maternal and Child Health Hospital

Lin-Qing Pan

Lianyungang Maternal and Child Health Hospital

Li-Sha Tang

Lianyungang Maternal and Child Health Hospital

Yu-Gui Cui

Nanjing Medical University

Jia-Yin Liu (D jyliu_nj@126.com )

First Affiliated Hospital $₫$ Nanjing Medical University

\section{Research}

Keywords: Recurrent miscarriage, ENO1, villous trophoblasts

Posted Date: August 26th, 2020

DOl: https://doi.org/10.21203/rs.3.rs-63653/v1

License: (c) (i) This work is licensed under a Creative Commons Attribution 4.0 International License.

Read Full License 


\section{Abstract}

Recurrent miscarriage (RM) is a common reproductive endocrine disease in women of childbearing age. At present, the etiology of RM in approximately 50\% women remains unknown. The purpose of this study was to explore the possible mechanism(s) of enolase 1 (ENO1) in RM and to seek a new target for clinical diagnosis and treatment of the disease. In this study, we detected the expression difference of ENO1 in villous tissues between the RM group and the control group, and we found that ENO1 was significantly lower in the RM group. Immunohistochemistry was also performed to examine the localization and expression of ENO1 in villous cytotrophoblast cells, and we found that ENO1 was mainly expressed in the cytoplasm, cell membrane, and nucleus of trophoblast cells. The villous trophoblast cell membrane coloration in the control group was significantly darker than that in the RM group, suggesting that ENO1 is expressed at low levels on the cell membrane of trophoblast cells of RM. EN01 knockdown significantly inhibited cell migration and proliferation compared with the blank control group. Therefore, we conclude that ENO1 may mediate the occurrence of RM by downregulating the proliferation and invasion of villous trophoblasts. The specific mechanism needs further clarification.

\section{Introduction}

Generally, recurrent miscarriage (RM) is defined as the failure of 3 or more consecutive clinical pregnancies before 20 weeks of gestation with the same partner. However, several reproductive medicine societies define RM as two or more consecutive pregnancy losses recognized under ultrasound or histopathology [1, 2]. According to the 2016 Chinese expert consensus and guidelines, RM refers to 3 or more consecutive spontaneous abortions before 28 weeks of pregnancy[3]. RM is a common reproductive disease worldwide, plaguing $2 \% \sim 5 \%$ of conceived women[4], and its incidence has exhibited an obvious increasing trend.

The known pathogenic causes of RM include couples or embryonic chromosomal abnormalities, prothrombotic state, uterine malformation, endocrine dysfunction, infection, and immune factors[2]. It is estimated that the etiology of RM in approximately $50 \%$ of women is unknown. Environmental, genetic, and epigenetic factors are potentially causal roles in RM[2,3]. Multiple studies have found that trophoblast invasion and spiral artery remodeling dysfunction are reduced in decidual tissues with RM. This feature is also considered to be one of the causes of RM, and it is also speculated to be a critical element to elucidate its pathogenesis.

Placental trophoblast cells play an important role in embryonic development and pregnancy maintenance; their two main functions are invasiveness and endocrine function. Trophoblast cell invasiveness is highly similar to that of tumor cells. Its normal proliferative capacity, migratory status, and invasive ability provide the basic conditions for the formation of a functional placenta, embryonic development, and pregnancy maintenance. Trophoblast cell invasiveness has strict spatiality and temporality, which is highly regulated, and is especially increased in early pregnancy but weakens or even disappears in mid-to-late pregnancy. The aggressive behavior of extravillous trophoblast (EVT) cells in the 
uterine epithelium is a prerequisite for placenta formation. Placental trophoblast cells have functional characteristics similar to cancer cells; both have a high degree of proliferation and invasion capabilities, and there are many common gene expression similarities in both cells[5].

Enolase (ENO), known as 2-phosphate-D-glycerol hydrolase, is a metabolic enzyme involved in pyruvate synthesis that maintains the level of ATP in cells and meets the energy required for life activities. ENO can exist in the cytoplasm, cell membrane and nucleus simultaneously[6]. Recent studies have shown that ENO1 plays an important regulatory role in many biological processes, such as plasminogen activation and plasmin activity, myogenesis, apoptosis, tumorigenesis and development[7]. The biological characteristics of trophoblasts and tumor cells are highly similar. The clear functional role of ENO1 is also closely related to the etiology of recurrent abortion. Whether ENO1 plays a critical role in the development mechanism of RM is worth exploring.

This article aims to investigate the expression of ENO1 in villous tissue of RM and the effect of the gene on the proliferation and invasion of villous trophoblasts.

\section{Material And Methods}

\subsection{Participating cohorts}

The inclusion criteria of the sample group $(n=8)$ of this study included women who experienced spontaneous abortion 3 or more times before 12 weeks of pregnancy. The ultrasound examination of these pregnancies confirmed that no primitive cardiac tube pulsation was found, and the pregnancy was terminated by induced abortion. Chromosomal abnormal villi were ruled out by array-CGH diagnosis. The control group $(n=8)$ included women selected at random who underwent abortion at the same hospital before 12 weeks of pregnancy without adverse pregnancy.

Villous tissues were obtained under aseptic conditions, washed thoroughly with sterile physiological saline to remove blood and decidual tissue, divided into $0.5 \mathrm{~cm}$ in diameter, and labeled in liquid nitrogen for storage.

\subsection{Cell line}

HTR-8/SVneo cells were obtained from the China Typical Culture Preservation Center (Wuhan University, China), which is an extracellular trophoblast cell line originating from first-trimester villous explants.

\subsection{Total RNA isolation and quantitative real-time polymerase chain reaction ( $q R T-P C R$ )}

Total RNA was extracted from villi tissue trophoblast cells using TRIzol reagent (Invitrogen, USA). cDNA was synthesized using a PrimeScript First Strand cDNA Synthesis Kit (TaKaRa, Japan), and qPCR was performed using SYBR Green I mix (Takara, Japan). The thermocycling conditions were as follows: $95^{\circ} \mathrm{C}$ 
for $10 \mathrm{~s}, 40$ cycles of $95^{\circ} \mathrm{C}$ for $5 \mathrm{~s}$ and $60^{\circ} \mathrm{C}$ for $30 \mathrm{~s}$. GAPDH mRNA was used as an internal standard, and each result was normalized to GAPDH. All experiments were performed in triplicate, and Ct values were converted to fold expression changes by the 2- $\triangle \mathrm{DCT}$ method. The following primers were used: GAPDH: 5'-AATCCCATCACCATCTTCC-3' (forward) and 5'-GAGTCCTTCCACGATACCAA-3' (reverse), ENO1: 5'-AAAGCTGGTGCCGTTGAGAA-3' and 5'-GGTTGTGGTAAACCTCTGCTC-3' (reverse).

\subsection{Immunohistochemistry}

Villi tissues were fixed with $4 \%$ paraformaldehyde solution, embedded in paraffin, and stained routinely. The sections were dewaxed in turn with xylene, dehydrated with gradient alcohol, and antigen-repaired by microwave drying in lemon saline for 15 minutes. The endogenous peroxidase activity of the sections was blocked by deparaffinization and $0.3 \%$ hydrogen peroxide treatment for 15 minutes. The sheep serum working solution was added dropwise to further seal the sections, and then the rabbit primary antibody (Abcam) against ENO1 was added dropwise and incubated at $4{ }^{\circ} \mathrm{C}$ for more than $16 \mathrm{~h}$. After washing, biotinylated secondary antibody (G1210-2-A, Servicebio) was added dropwise and incubated for 60 minutes at room temperature. The expression of EN01 was visualized by 3,30-diaminobenzidine tetrahydrochloride (DAB) section staining. After the slices were developed, they were counterstained with hematoxylin. Analysis was performed after taking pictures under a fluorescence microscope (Nikon, Japan).

\subsection{In vitro experiments}

HTR-8/SVneo cells were cultured in RPMI 1640 medium with 10\% fetal bovine serum (FBS), $100 \mu \mathrm{g} / \mathrm{ml}$ penicillin-streptomycin and $100 \mu \mathrm{g} / \mathrm{ml}$ penicillin (all Gibco, USA) at $37^{\circ} \mathrm{C}$ in a $5 \% \mathrm{CO} 2$ incubator.

At 36 h, 48 h, 72 h, and 96 h after transfection with EN01 siRNA, cells were harvested for cell proliferation and invasion analysis. For cell proliferation analysis, cells were seeded into 96-well plates (5000 cells per well). After transfection, $10 \mu$ CCK8 solution (Dojindo, Japan) was added into each well and cultured for $1 \mathrm{~h}$, and the absorbance was measured at $490 \mathrm{~nm}$.

The proliferation capacity of HTR-8/SVneo cells after EN01-siRNA transfection was determined by a clonogenic assay. Cells at the logarithmic growth phase were seeded in 6-well plates and cultured in a $37^{\circ} \mathrm{C}$ incubator for $24 \mathrm{~h}$. A single cell suspension was prepared for EN01-siRNA transfection. Twenty-four hours after transfection, the cell concentration was trypsinized and adjusted to $1 \times 10^{5}$ cells. Following incubation for $10 \sim 14$ days, the cells were fixed with 4\% paraformaldehyde in PBS for 10 minutes and stained with crystal violet solution (1\% solution; Sigma-Aldrich) for 60 minutes. After washing with PBS again, the number of clones was counted and recorded.

For invasion analysis, $500 \mu \mathrm{l}$ medium containing 10\% FBS was added per well in a 24-well plate. Transwell chamber inserts were put into the wells with $100 \mu$ l cell suspension mixed with serum-free medium. The cells were immobilized with polyoxymethylene and photographed after $36 \mathrm{~h}$.

\subsection{Western blot analysis}


Total protein was extracted from villous tissues and HTR-8/SVneo cells using RIPA buffer and a protease inhibitor and quantified by the bicinchoninic acid method (Biyuntian, China). Western blots were performed using antibodies against ENO1 (1:2000, Abcam, UK), GAPDH (1:10000, Proteintech, USA), and goat anti-rabbit secondary antibodies (1:500, Proteintech, USA) following standard protocols. Sixty micrograms of protein were subjected to SDS-PAGE and transferred to PVDF membranes. The PVDF membrane was placed in TBST containing $5 \%$ skimmed dried milk and sealed in a shaker at $37^{\circ} \mathrm{C}$ for $1 \mathrm{~h}$. Primary antibody was added followed by incubation at $4{ }^{\circ} \mathrm{C}$ overnight, and then the membranes were washed with TBST three times and incubated with HRP-conjugated secondary antibody for $1 \mathrm{~h}$ at $37^{\circ} \mathrm{C}$. The immunoreactive bands were visualized using ECL-Plus reagent (Biyuntian, China). Three repetitions were performed. The intensities of the protein bands were quantitated using ImageJ software.

\subsection{Statistical analysis}

All experiments were repeated 3 times. The data were analyzed by IBM SPSS17 for Windows software. The experimental data had homogeneous variance, expressed as the mean \pm SD. Independent sample $T$ tests were used to perform pairwise comparisons between the two groups. The difference was statistically significant at a $\mathrm{P}$ value of less than 0.05 .

\section{Results}

\subsection{Expression of ENO1 in villi of recurrent miscarriage patients}

Real-time RT-PCR and Western blotting were used to detect the expression level of ENO1 in the villous tissue of RM patients and the control group. The mRNA and protein levels of ENO1 were significantly lower in RM tissues than in controls, decreasing by $29.7 \%$ and $70.9 \%$, respectively, with statistical significance $(P=0.0118, P=0.0079)$ (Fig. 1).

To further explore the function of ENO1, the cellular localization and protein expression of ENO1 were measured using immunohistochemistry. The results showed that ENO1 was mainly expressed in the cytoplasm, cell membrane and nucleus of trophoblast cells. The cell membranes of villous trophoblast cells in the control group were dark brown, while those of villous trophoblast cells in patients with RM appeared light yellow (Fig. 2).

\subsection{Knockdown of ENO1 inhibited the proliferation and invasion of HTR-8/SVneo cells}

EN01 siRNA interference fragments were transfected in vitro, and three ENO1 siRNAs and one nonspecific ENO1 siRNA fragment were designed for different sites. The knockdown efficiency of ENO1 was detected by Western blot analysis. As shown in Fig. 3A, ENO1 protein levels decreased by $85 \%$ and $87 \%$ after transfection with siRNA1 and siRNA2, respectively, in HTR-8/SVneo cells but only decreased by $30 \%$ after transfection with siRNA3. Therefore, ENO1 siRNA1 and siRNA2 were selected for the follow-up experiment 
(Fig. 3A). The CCK8 results showed that the cell activity did not change significantly after transfection of EN01 siRNA1 and EN01 siRNA2 for $72 \mathrm{~h}$. However, after $96 \mathrm{~h}$ of transfection, the cell proliferation ability decreased by $22.9 \%$ and $25.9 \%$ compared with the control group $(P=0.0116, P=0.0126)$ (Fig. 3B). The results of clone formation experiments revealed that transfection of EN01 siRNA1 and ENO1 siRNA2 significantly inhibited the cell activity of villous trophoblast cells by $46.1 \%$ and $76.6 \%(P=0.0003, P<$ 0.0001), respectively, compared with the control group (Fig. 4A). The Transwell chamber was used to detect the effect of ENO1 knockdown on the invasion ability of trophoblast cells. The results demonstrated that after $36 \mathrm{~h}$ of treatment with ENO1 siRNA1 and ENO1 siRNA2, the number of villous trophoblast cells that passed through Matrigel was significantly reduced, which was $43.1 \%$ and $54.2 \%$ lower than the control group $(P=0.0056, P=0.0036)$, indicating that ENO1 significantly inhibited the invasion ability of villous trophoblast cells (Fig. 4B).

\section{Discussion}

ENO is present in almost all mature tissues of the human body and coexists in the cytoplasm, cell membrane and nucleus[6]. ENO1, also known as a-enolase, is one of the most important enzymes in enolase. Recent studies have shown that ENOI plays a critical regulatory role in biological processes such as plasminogen activation, plasmin activity, myogenesis, apoptosis and tumor development[7]. ENO1 can also be used as an autoantigen to induce an immune inflammatory response[8]. The autoimmune response against ENO1 may be an important mechanism in diseases such as Hashimoto encephalopathy and glomerulonephritis[9]. EN01 may also play a crucial role in the stress response, which is important for cell survival. For example, myocardial cell damage could activate ENO1 in the cytoplasm, and then ENO1 is transported to the cytoskeleton and contractile filaments[10]. As a molecular companion of ENO1, the above structures are stabilized, and the normal function of the myocardium is maintained. Recently, substantial evidence has shown that ENO1 is related to the occurrence and metastasis of malignant tumors, such as metastatic breast cancer, pancreatic cancer, liver cancer and other cells with high malignancy. In pancreatic ductal adenocarcinoma (PDA) cells, ENO1 can act as a plasminogen receptor and promote cell invasion and migration. In addition, ENO1 silencing can induce oxidative stress and alter the metabolism of PDA cells. The results of the study suggest that ENO1 promotes the survival, migration, and transfer of PDA through a synergistic effect with integrin and urokinase plasminogen activator receptor (UPAR)[11]. The a-enolase on the surface of lung cancer cells promotes the degradation of extracellular matrix and the invasion of cancer cells. Targeting surface aenolase is a promising method for inhibiting tumor metastasis[12]. In addition, overexpression of ENO1 will increase the secretion of progesterone in granulosa cells cultured in vitro in goose follicles, suggesting that it may be related to corpus luteum function[13].

The role of ENO1 in RM and its mechanism of action in trophoblasts is unclear. The related functions of ENO1 that are known, including plasminogen activity, endocrine activity, autoimmune response, and oncogene promoting cell migration, invasion and metastasis, are closely related and highly similar to the molecular mechanisms of RM. 
Using real-time RT-PCR and Western blot experiments, we found that the mRNA and protein levels of ENO1 in the villous tissue of patients with RM were significantly lower than those of normal abortions. Through immunohistochemical experiments, we found that ENO1 was expressed in the cytoplasm, cell membrane and nucleus of trophoblast cells. The villous trophoblast cell membrane coloration in the control group was significantly darker than that in the RM group, suggesting that ENO1 is expressed at low levels on the cell membrane of trophoblast cells of patients with RM. The villous trophoblast cell membrane coloration was significantly deeper than that in the recurrent abortion group, suggesting that ENO1 is expressed at low levels on the cell membrane of villous trophoblast cells. Previous studies have found that ENO1 located on the surface of cell membranes plays an important role in the plasmin system and is a plasminogen-binding molecule[7]. The interaction between plasmin and ENO1 promotes the pathophysiological process of cell migration, such as inflammation, cell invasion and cancer metastasis.

The implantation process of the embryo depends on the dynamic balance between the synthesis of the placental trophoblast cells and the secretion of a large number of fibrinolysis/anti-fibrinolysis-related protein factors, which can effectively dissolve the endometrial tissue to facilitate the invasion of trophoblast cells and the formation of the placenta while causing local bleeding of decidual tissue and leading to adverse pregnancy outcomes. We found that the expression of ENO1 is downregulated on the cell membrane of trophoblast cells in RM. According to the previous function of ENO1, ENO1 may reduce the invasion and migration of trophoblast cells by inhibiting the action of the fibrinolytic system, thereby promoting the occurrence of RM, which needs further experimental verification.

Trophoblast cell dysfunction can lead to pregnancy-related diseases such as intrauterine growth restriction, preeclampsia, eclampsia, spontaneous abortion, premature delivery, and choriocarcinoma[14]. We detected changes in the proliferation and invasion function of HTR-8/SVneo cells after knocking down ENO1 through CCK8 and Transwell chamber assays. We found that the proliferation and invasion function of EVT cells decreased significantly with the downregulation of ENO1, which is consistent with the biological behavior of tumor cells. Fu QF et al. found that upregulation of ENO1 activates FAK/PI3K/AKT and its downstream signals, regulating glycolysis, the cell cycle and EMT-related genes to promote the proliferation and metastasis of non-small cell lung cancer (NSCLC)[15]. Overexpression of ENO1 is also related to the progression of gliomas, and inhibiting ENO1 expression can inhibit the process of cell growth, migration and invasion by inactivating the PI3K/Akt pathway in glioma cells[16]. In previous studies on RM, cell proliferation, invasion, migration, and angiogenesis-related signaling pathways, such as the Wnt/ $\beta$-catenin signaling pathway[17], the PI13K signaling pathway[18], and the ERK and other signaling pathways[19], participated in the occurrence and development of RM. Further research is needed to verify whether ENO1 affects the function of trophoblast cells through the above pathways.

In summary, the low expression of ENO1 in tissues of RM and the effect on the function of EVT cells in vitro suggest that it may play an important role in RM, and further studies of the specific mechanism are needed. Whether ENO1 leads to trophoblast cell proliferation and invasion by activating plasminogen and 
whether ENO1 is involved in the occurrence of recurrent miscarriage through the autoimmune response and affects the secretion of progesterone and the related mechanisms are worthy of further discussion.

\section{Declarations}

\section{Conflicts of interest}

The authors declare they have no competing interests.

\section{Consent for publication}

The author declares that all work described here has not been published before (except in the form of an abstract or as part of a published lecture, review, or thesis); that its publication has been approved by all co-authors.

\section{Ethical approval}

This study was approved by the Ethics Committee of Lianyungang Maternal and Child Health Hospital (No. LYG-ME2017003). All individual participants signed informed consent.

\section{Availability of data and material}

All data generated or analyzed during this study are included in this published article

\section{Funding}

This work was supported by the National Key Research and Development Program of China (2017YFC1001303, 2016YFC1000603) and National Nature and Science Fundation of China (81730041), and a program of Jiangsu Province Health Department (YXZXB2016001). Jiangsu Basic Research Program (Natural Science Foundation, BK20161298)『Jiangsu Commission of Health (H2018014), and 521 projects of Lianyungang city (LYG52105-2018080).

\section{Authors' contribution}

Jiayin Liu and Yugui Cui designed experiment and reviewed the manuscript. Huaiyun Tang and Linqing Pan performed research. Lisha Tang contributed new reagents. Huaiyun Tang and Linqing Pan analyzed data and wrote the paper.

\section{Acknowledgments}

The authors are very grateful to Li Gao and Chao Gao, who work in the State Key Laboratory of Reproductive Medicine, for their technical assistance.

\section{References}


1. M. Practice Committee of American Society for Reproductive, Definitions of infertility and recurrent pregnancy loss: a committee opinion, Fertil Steril 99 (2013) 63. 10.1016/j.fertnstert.2012.09.023.

2. E.G.G.o. RPL, R. Bender Atik, O.B. Christiansen, J. Elson, A.M. Kolte, S. Lewis, S. Middeldorp, W. Nelen, B. Peramo, S. Quenby, N. Vermeulen, M. Goddijn, ESHRE guideline: recurrent pregnancy loss, Hum Reprod Open 2018 (2018) hoy004. 10.1093/hropen/hoy004.

3. L.X. Wang, L.H. Hou, Y.M. Xie, K. Ma, S.L. Sun, Z. Jin, H.L. Du, D.M. Wang, H. Zhao, Y.F. Liu, L. Tang, K.Y. Shu, C.Z. Zhang, W. Shi, S.Y. Zhan, J.P. Liu, W. Chen, Y.L. Chen, [Expert consensus statement on Kangfu Xiaoyan Suppository in treatment of pelvic inflammatory in clinical practice], Zhongguo Zhong Yao Za Zhi 44 (2019) 4350-4353. 10.19540/j.cnki.cjcmm.20190726.501.

4. H. El Hachem, V. Crepaux, P. May-Panloup, P. Descamps, G. Legendre, P.E. Bouet, Recurrent pregnancy loss: current perspectives, Int J Womens Health 9 (2017) 331-345. 10.2147/IJWH.S100817.

5. C.A. Mullen, Review: analogies between trophoblastic and malignant cells, Am J Reprod Immunol 39 (1998) 41-49. 10.1111/j.1600-0897.1998.tb00332.x.

6. V. Pancholi, V.A. Fischetti, alpha-enolase, a novel strong plasmin(ogen) binding protein on the surface of pathogenic streptococci, J Biol Chem 273 (1998) 14503-14515. 10.1074/jbc.273.23.14503.

7. P. Caturegli, A. De Remigis, N.R. Rose, Hashimoto thyroiditis: clinical and diagnostic criteria, Autoimmun Rev 13 (2014) 391-397. 10.1016/j.autrev.2014.01.007.

8. H. Liu, Z. Shan, C. Li, J. Mao, X. Xie, W. Wang, C. Fan, H. Wang, H. Zhang, C. Han, X. Wang, X. Liu, Y. Fan, S. Bao, W. Teng, Maternal subclinical hypothyroidism, thyroid autoimmunity, and the risk of miscarriage: a prospective cohort study, Thyroid 24 (2014) 1642-1649. 10.1089/thy.2014.0029.

9. G. Kocak, B. Huddam, A. Azak, L. Ortabozkoyun, M. Duranay, Coexistent findings of renal glomerular disease with Hashimoto's thyroiditis, Clin Endocrinol (Oxf) 76 (2012) 759-762. 10.1111/j.13652265.2011.04302.x.

10. Y. Mizukami, A. Iwamatsu, T. Aki, M. Kimura, K. Nakamura, T. Nao, T. Okusa, M. Matsuzaki, K. Yoshida, S. Kobayashi, ERK1/2 regulates intracellular ATP levels through alpha-enolase expression in cardiomyocytes exposed to ischemic hypoxia and reoxygenation, J Biol Chem 279 (2004) 5012050131. 10.1074/jbc.M402299200.

11. M. Principe, S. Borgoni, M. Cascione, M.S. Chattaragada, S. Ferri-Borgogno, M. Capello, S. Bulfamante, J. Chapelle, F. Di Modugno, P. Defilippi, P. Nistico, P. Cappello, C. Riganti, S. Leporatti, F. Novelli, Alpha-enolase (ENO1) controls alpha v/beta 3 integrin expression and regulates pancreatic cancer adhesion, invasion, and metastasis, J Hematol Oncol 10 (2017) 16. 10.1186/s13045-0160385-8.

12. K.C. Hsiao, N.Y. Shih, H.L. Fang, T.S. Huang, C.C. Kuo, P.Y. Chu, Y.M. Hung, S.W. Chou, Y.Y. Yang, G.C. Chang, K.J. Liu, Surface alpha-enolase promotes extracellular matrix degradation and tumor metastasis and represents a new therapeutic target, PLoS One 8 (2013) e69354. 10.1371/journal.pone.0069354.

13. Y. Ye, C. Kuhn, M. Kosters, G.J. Arnold, H. Ishikawa-Ankerhold, C. Schulz, N. Rogenhofer, C.J. Thaler, S. Mahner, T. Frohlich, U. Jeschke, V. von Schonfeldt, Anti alpha-enolase antibody is a novel 
autoimmune biomarker for unexplained recurrent miscarriages, EBioMedicine 41 (2019) 610-622. 10.1016/j.ebiom.2019.02.027.

14. Q. Zhang, S. Yu, X. Huang, Y. Tan, C. Zhu, Y.L. Wang, H. Wang, H.Y. Lin, J. Fu, H. Wang, New insights into the function of Cullin 3 in trophoblast invasion and migration, Reproduction 150 (2015) 139-149. 10.1530/REP-15-0126.

15. Q.F. Fu, Y. Liu, Y. Fan, S.N. Hua, H.Y. Qu, S.W. Dong, R.L. Li, M.Y. Zhao, Y. Zhen, X.L. Yu, Y.Y. Chen, R.C. Luo, R. Li, L.B. Li, X.J. Deng, W.Y. Fang, Z. Liu, X. Song, Alpha-enolase promotes cell glycolysis, growth, migration, and invasion in non-small cell lung cancer through FAK-mediated PI3K/AKT pathway, J Hematol Oncol 8 (2015) 22. 10.1186/s13045-015-0117-5.

16. Y. Song, Q. Luo, H. Long, Z. Hu, T. Que, X. Zhang, Z. Li, G. Wang, L. Yi, Z. Liu, W. Fang, S. Qi, Alphaenolase as a potential cancer prognostic marker promotes cell growth, migration, and invasion in glioma, Mol Cancer 13 (2014) 65. 10.1186/1476-4598-13-65.

17. H. Xiang, H. Yan, B. Sun, F. Feng, P. Chen, Decreased expression of long non-coding RNA SNHG7 cause recurrent spontaneous abortion through suppression proliferation and invasion of trophoblast cells via miR-34a, Am J Transl Res 11 (2019) 463-472.

18. S. Qin, Y. Zhang, J. Zhang, F. Tian, L. Sun, X. He, X. Ma, J. Zhang, X.R. Liu, W. Zeng, Y. Lin, SPRY4 regulates trophoblast proliferation and apoptosis via regulating IFN-gamma-induced STAT1 expression and activation in recurrent miscarriage, Am J Reprod Immunol 83 (2020) e13234. 10.1111/aji.13234.

19. X. Liu, J. Zhao, X. Luan, S. Li, J. Zhai, J. Liu, Y. Du, SPARCL1 impedes trophoblast migration and invasion by down-regulating ERK phosphorylation and AP-1 production and altering EMT-related molecule expression, Placenta 89 (2020) 33-41. 10.1016/j.placenta.2019.10.007.

\section{Figures}


A

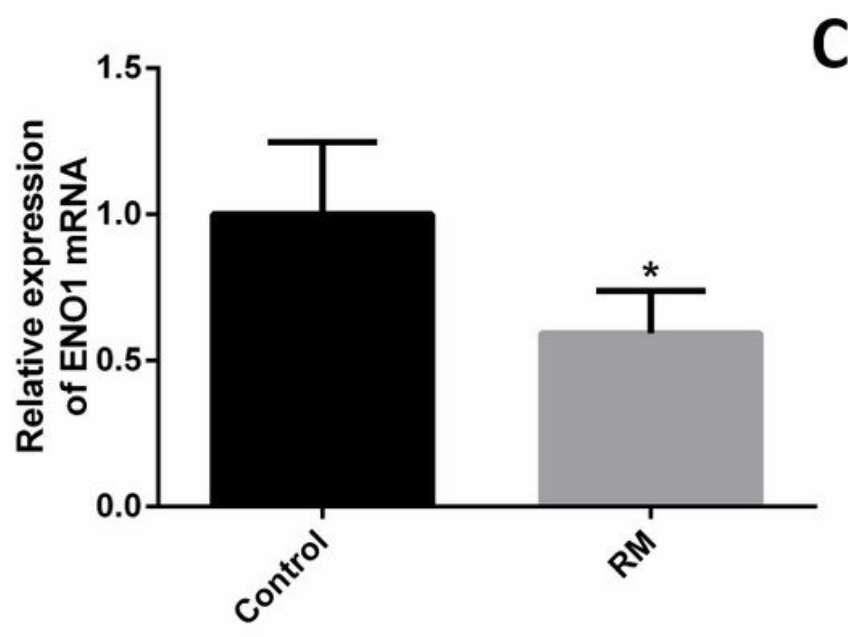

C

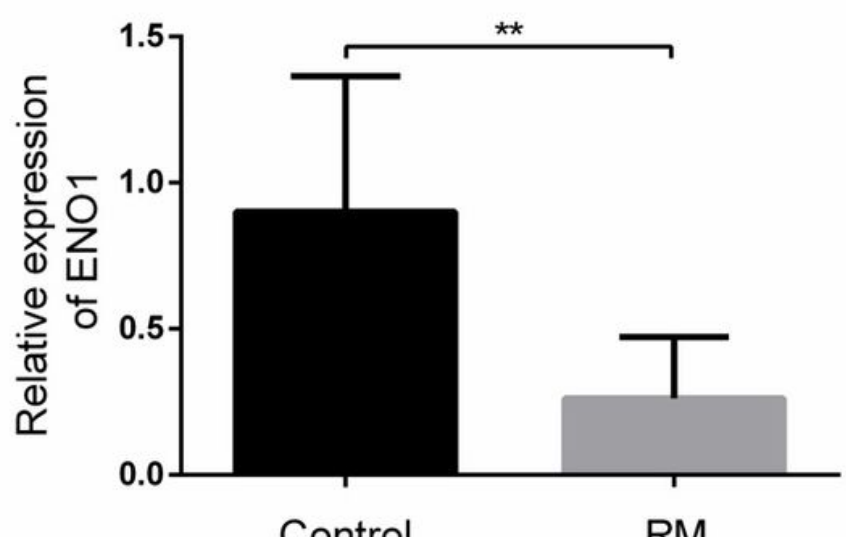

Control

RM

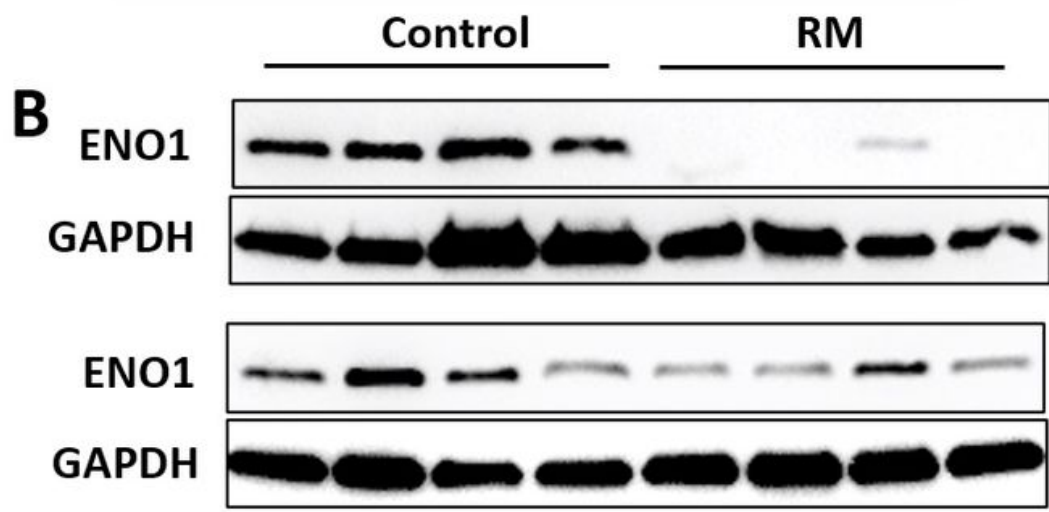

Figure 1

A. EN01 mRNA level in villous tissue of patients with recurrent miscarriage was $29.7 \%$ lower than that of the control group $(P=0.0118) ; B, C$. EN01 protein level in villous tissue of patients with recurrent miscarriage was $70.9 \%$ lower than that of the control group $(P=0.0079)$ * $P \varangle 0.05$, ** $P \otimes 0.01$ 


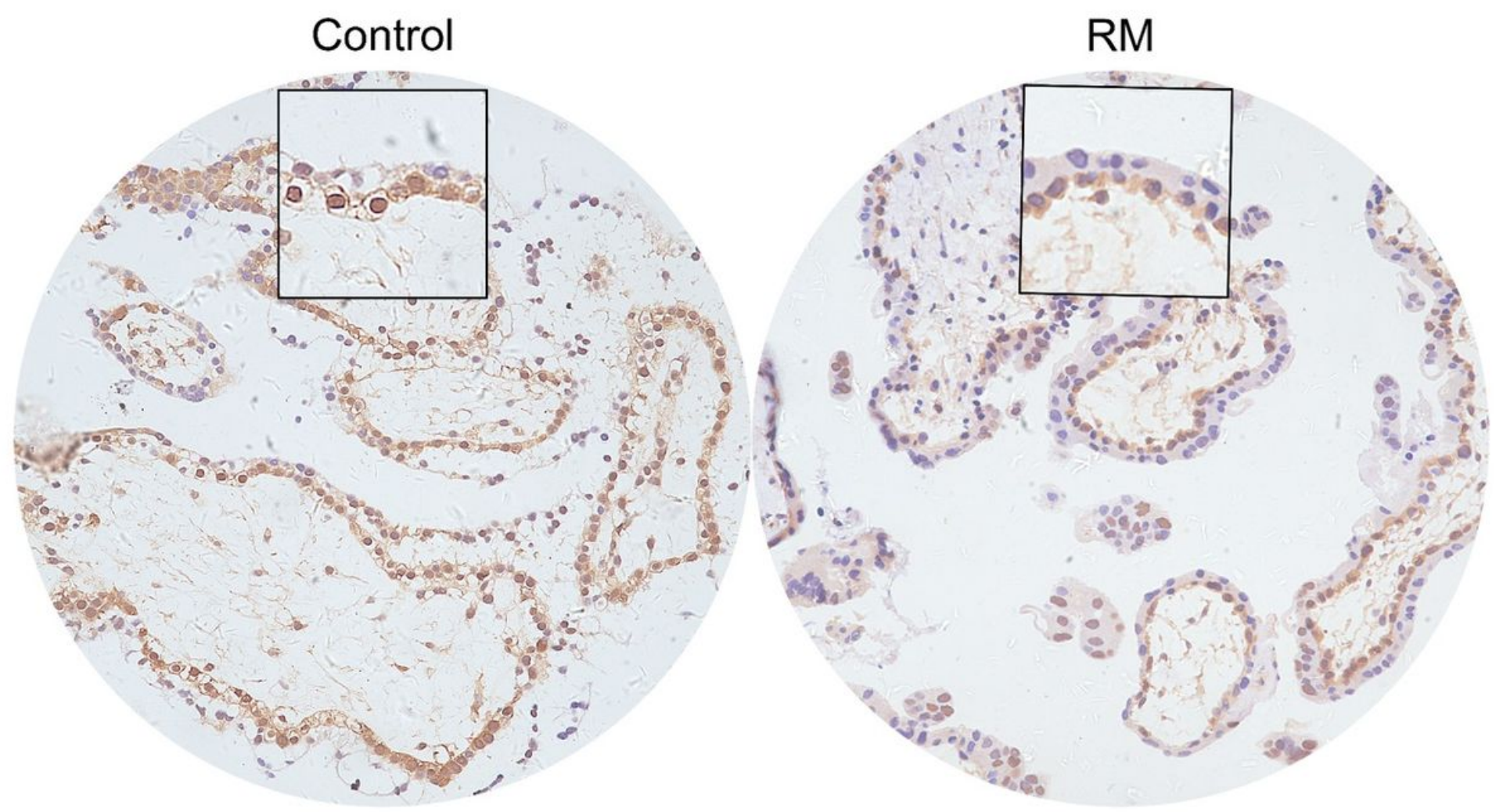

Figure 2

ENO1 localization and expression in trophoblast cells. Immunohistochemistry analysis of ENO1 stain from placental villus of RM patients and health control women. The cell membranes of villous trophoblast cells in the control group were dark brown, while those of villous trophoblast cells in patients with RM appeared light yellow.

A

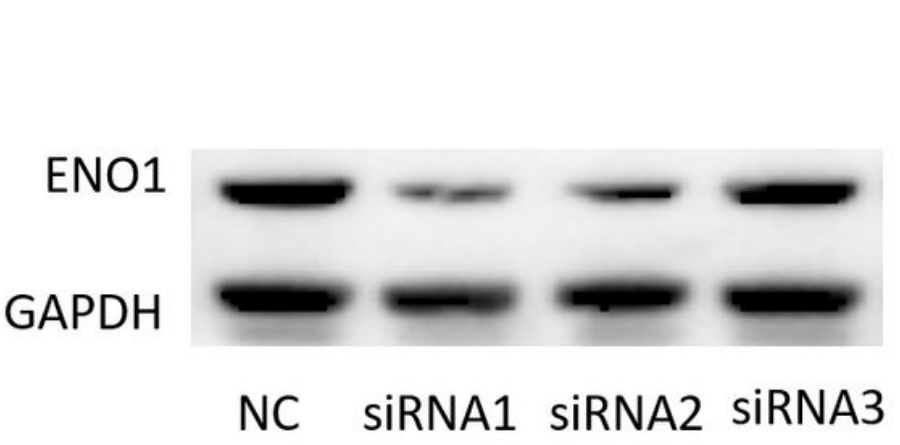

B

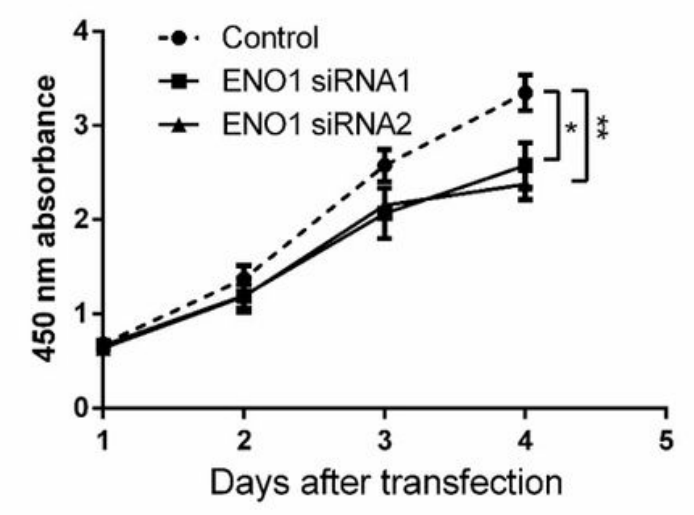

Figure 3

A. Western blot analysis was used to detect the knockdown efficiency of three ENO1 siRNAs in HTR8/SVneo cells. After transfection of ENO1 siRNA1 and siRNA2, ENO1 protein expression was downregulated by $85 \%$ and $87 \%$ for subsequent experiments; B. CCK 8 assays were performed to determine cell proliferation of HTR-8/SVneo cells after transfection with ENO1 siRNA. As revealed by the 
results, the transfection of ENO1 siRNA1 and EN01 siRNA2 for $96 \mathrm{~h}$ reduced the cell proliferation ability by $22.9 \%$ and $25.9 \%$, respectively, compared with the control group $(P=0.0116, P=0.0126)$. $P \llbracket 0.05 \nabla^{\star \star} P$

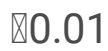

A

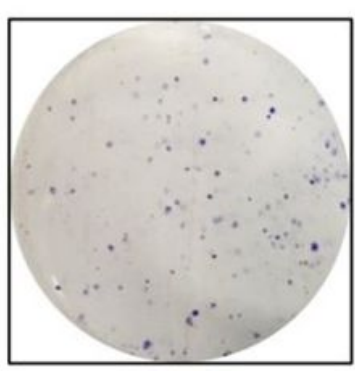

NC

C

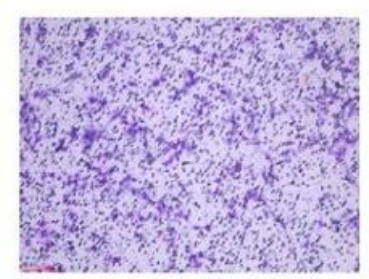

NC
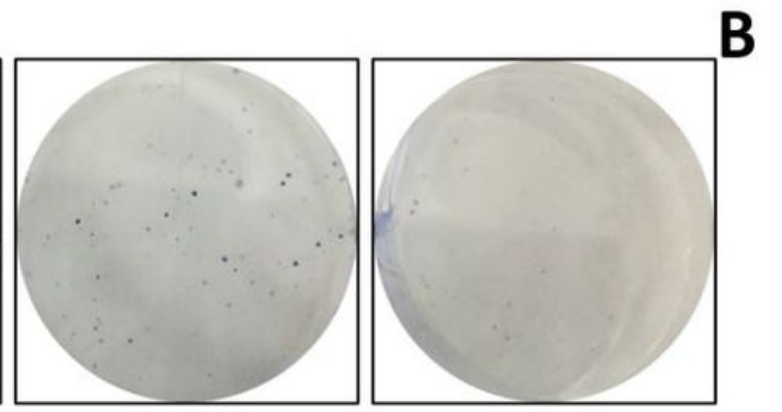

siRNA2

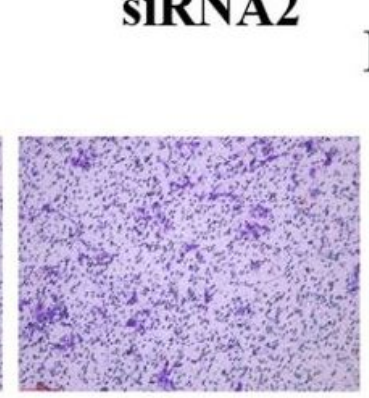

SIRNA2

D
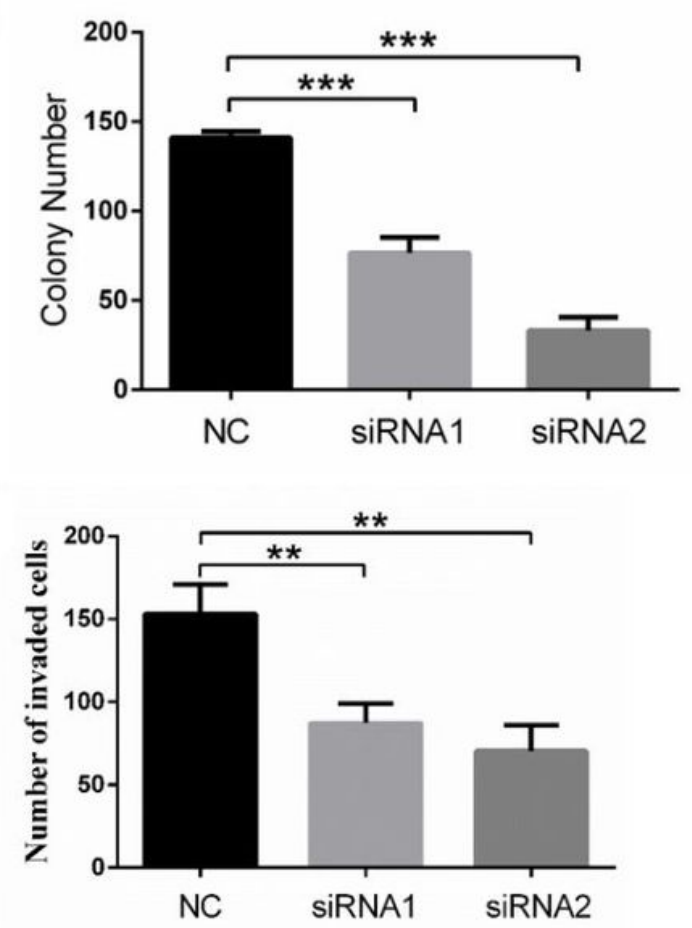

\section{Figure 4}

Quantification and image of the clonogenic assay and Transwell chamber assay after HTR-8/SVneo cell transfection with ENO1 siRNA. A and B. The clonogenic results showed that compared with the control group, ENO1 siRNA1 and ENO1 siRNA2 transfection significantly inhibited the cell activity of villous trophoblast cells, which were downregulated by $46.1 \%$ and $76.6 \%$, respectively $(P=0.0003, P<0.0001)$. C and $\mathrm{D}$. The Transwell chamber assay results showed that after $36 \mathrm{~h}$ of transfection with ENO1 siRNA1 and ENO1 siRNA2, the number of villous trophoblast cells that passed through Matrigel decreased by $43.1 \%$ and $54.2 \%$, respectively $(P=0.0056, P=0.0036)$. ${ }^{*} P \otimes 0.01, * \star * P<0.001$. 